\title{
Enhancing the bioactivity of Poly(lactic-co-glycolic acid) scaffold with a nano-hydroxyapatite coating for the treatment of segmental bone defect
} in a rabbit model

\author{
This article was published in the following Dove Press journal: \\ International Journal of Nanomedicine \\ 8 May 2013 \\ Number of times this article has been viewed
}

\author{
De-Xin Wang ${ }^{1, *}$ \\ Yao $\mathrm{He}^{2, *}$ \\ Long $\mathrm{Bi}^{1, *}$ \\ Ze-Hua $\mathrm{Qu}^{2}$ \\ Ji-Wei Zou' \\ Zhen $\operatorname{Pan}^{2}$ \\ Jun-Jun Fan' \\ Liang Chen ${ }^{2}$ \\ Xin Dong' \\ Xiang-Nan Liu² \\ Guo-Xian Pei' \\ Jian-Dong Ding ${ }^{2}$ \\ 'Department of Orthopaedics, Xijing \\ Hospital, The Fourth Military Medical \\ University, Xi'an, People's Republic \\ of China; ${ }^{2}$ State Key Laboratory of \\ Molecular Engineering of Polymers, \\ Department of Macromolecular \\ Science, Fudan University, Shanghai, \\ People's Republic of China \\ *These authors contributed equally to \\ this work
}

Correspondence: Guo-Xian Pei Department of Orthopaedics, Xijing Hospital, The Fourth Military Medical University, 127 West Changle Road,

Xi'an, Shaanxi 710032 ,

People's Republic of China

Tel +862984773524

Email guoxianpei_fmmu@।63.com

Jian-Dong Ding

State Key Laboratory of Molecular

Engineering of Polymers, Department

of Macromolecular Science,

Fudan University, Shanghai 200433,

People's Republic of China

Tel +86 2l 65643506

Email jdding I@fudan.edu.cn
Purpose: Poly(lactic-co-glycolic acid) (PLGA) is excellent as a scaffolding matrix due to feasibility of processing and tunable biodegradability, yet the virgin scaffolds lack osteoconduction and osteoinduction. In this study, nano-hydroxyapatite (nHA) was coated on the interior surfaces of PLGA scaffolds in order to facilitate in vivo bone defect restoration using biomimetic ceramics while keeping the polyester skeleton of the scaffolds.

Methods: PLGA porous scaffolds were prepared and surface modification was carried out by incubation in modified simulated body fluids. The nHA coated PLGA scaffolds were compared to the virgin PLGA scaffolds both in vitro and in vivo. Viability and proliferation rate of bone marrow stromal cells of rabbits were examined. The constructs of scaffolds and autogenous bone marrow stromal cells were implanted into the segmental bone defect in the rabbit model, and the bone regeneration effects were observed.

Results: In contrast to the relative smooth pore surface of the virgin PLGA scaffold, a biomimetic hierarchical nanostructure was found on the surface of the interior pores of the nHA coated PLGA scaffolds by scanning electron microscopy. Both the viability and proliferation rate of the cells seeded in nHA coated PLGA scaffolds were higher than those in PLGA scaffolds. For bone defect repairing, the radius defects had, after 12 weeks implantation of nHA coated PLGA scaffolds, completely recuperated with significantly better bone formation than in the group of virgin PLGA scaffolds, as shown by X-ray, Micro-computerized tomography and histological examinations.

Conclusion: nHA coating on the interior pore surfaces can significantly improve the bioactivity of PLGA porous scaffolds.

Keywords: PLGA, nano-hydroxyapatite, bone tissue engineering, BMSCs, bone defect

\section{Introduction}

Severe trauma, non-union fractures, and tumor resections can cause substantial clinical burden. For bone defect repairing, three approaches are typically used in the clinic: autograft bone, allograft bone, and artificial bone grafting materials. The use of allografts can avoid the associated operation for obtaining the autografts, but it is burdened by potential immune rejection, disease transmission, and decreased donor tissue sources. When considering safety, therapeutic efficacy, and lack of immune reaction, autologous bone grafting is the gold standard. ${ }^{1}$ However, this requires two separate surgical operations and has a risk of wound dehiscence, vessel injuries, hematomas, and infections. ${ }^{2}$ Bone tissue engineering has opened new possibilities to solve these problems; this technique is not only available in different shapes and sizes, but also stimulates rapid bone healing. 
An effective bone tissue engineering strategy requires appropriate scaffolds, which have good biocompatibility, suitable degradation rate, macroporous structure for cell growth and early vascularization, and osteoinductive properties. ${ }^{3}$ Poly(lactic-co-glycolic acid) (PLGA) is a copolymer of lactide and glycolide and is a good candidate material. By changing the molar ratios of lactide and glycolide in the copolymer, the mechanical property and degradation rate can be adjusted to fit various biomedical applications. ${ }^{4-6}$ While PLGA scaffolds exhibit many advantages, such as good processibility and tunable biodegradation rate, lack of osteoconduction and osteoinduction partially hinders their application in bone tissue engineering. It is thus meaningful to introduce osteoconductive or osteoinductive inorganics into PLGA scaffolds.

While mixing inorganics with polymers has been previously used in tissue engineering and tissue repairing, ${ }^{7-10}$ the present work concerns a surface modification of polymeric porous scaffolds by biomimetic inorganics. The surface characteristics of biomaterials can influence cell-material interactions and eventual osteointegration. ${ }^{11}$ The interactions between cells and nano-topographies have attracted attention especially in recent years, and these interactions can be employed to promote cell functions. ${ }^{12}$ Natural tissues are of hierarchical structures with nano-, micro-, and macro-scale building blocks. Implants that have a hierarchical structure of micro/nanoscale surfaces might be more effective because they simulate natural structures. Some scaffold possessing these micro/nanostructures, have been proven to be efficient as tissue engineering scaffolds. ${ }^{13,14}$

In this study, we used nanohydroxyapatite (nHA)-coated PLGA scaffolds with a biomimetic surface chemistry. The coating was achieved by immersing virgin porous scaffolds in simulated body fluids (SBF), which led to precipitation of nHA on the interior surfaces of the porous scaffolds. However, a naïve method cannot guarantee a tight binding of the inorganic coating layer onto the main matrix. Therefore, we pretreated the scaffolds with oxygen plasma and then coated the interior surfaces of the porous scaffolds with nHA. Both in vitro and in vivo experiments were performed to analyze the biological performance of virgin PLGA scaffolds and nHA coated PLGA scaffolds. Autologous bone marrow stromal cells (BMSCs) from rabbits were used as seeding cells, and New Zealand rabbits were used as the animal model. The results showed that the nHA coated PLGA scaffolds have improved biocompatibility and have more efficient capabilities for bone defect healing.

\section{Materials and methods Fabrication of PLGA and nHA coated PLGA scaffolds}

Commercial PLGA (85:15 molar ratio of lactide/glycolide) was purchased from Purac Biochem (Gorinchem, The Netherlands). The porous PLGA scaffolds were prepared using the room temperature molding/particle leaching method. ${ }^{15-18}$ Briefly, PLGA granules were dissolved in dichloromethane (10\% weight/volume) and mixed with a sieved sodium chloride particle of size $300-450 \mu \mathrm{m}$. The mixture was compressed into a mold with an interior size of $4 \mathrm{~mm}$ in diameter and $15 \mathrm{~mm}$ in height. After molding for 24 hours under pressure, the samples were taken out and laid in the fume hood for further evaporation of the solvent for another 24 hours. Next, the samples were washed to leach out sodium chloride particles in ion free water. After no $\mathrm{Cl}^{-}$ions in the leaching water were detected by $\mathrm{AgNO}_{3}$ solution indicating that all the particles were washed out, the porous scaffolds were desiccated and kept in a vacuum dryer before use.

Oxygen plasma pretreatment (DT-03, Suzhou OPS Plasma Technology Co. Ltd, Jiangsu, People's Republic of China) was employed to enhance the scaffold surface hydrophilicity and ion anchorage in future coating processes. The plasma chamber was vacuumized to $8 \mathrm{~Pa}$, thereafter filled with oxygen at a flow rate of 50 standard-state cubic centimeter per minute $(\mathrm{sccm})$. After the chamber pressure was stabilized, we performed the plasma treatment at $50 \mathrm{~W}$ for 8 minutes. The plasma-treated scaffolds were further exposed to the oxygen atmosphere in the chamber for another 5 minutes before they were removed.

SBF is comprised of similar components as human blood plasma $\left(\mathrm{Na}^{+} 142.0, \mathrm{~K}^{+} 5.0, \mathrm{Mg}^{2+} 1.5, \mathrm{Ca}^{2+} 2.5, \mathrm{Cl}^{-}\right.$103.0, $\left.\mathrm{HCO}_{3}^{-} 27.0, \mathrm{HPO}_{4}{ }^{2-} 1.0, \mathrm{SO}_{4}{ }^{2-} 0.5 \mathrm{mmol} / \mathrm{L}\right) .{ }^{19}$ In order to accelerate the $\mathrm{HA}$ formation in $\mathrm{SBF}$, a modified $\mathrm{SBF}$ of 10 -fold concentration adjusted with $\mathrm{HCl}$ to a $\mathrm{pH} 4-5$ was used in this study. Our modified SBF was filtered through a Whatman Klari-Flex ${ }^{\mathrm{TM}}$ system $(0.22 \mu \mathrm{m}$; GE Healthcare Bio-Sciences, Mississauga, ON, Canada) to remove impurities before use.

After the oxygen plasma pretreatment, the scaffolds were immersed in the modified SBF immediately, and vacuumed to ensure that pores were filled with the solution. The incubation was kept at room temperature for 12 hours. Thereafter, incubation was kept in a water bath at $37^{\circ} \mathrm{C}$, and the coating process was triggered by adding $\mathrm{NaHCO}_{3}$ to adjust to a pH 6-7. Incubation lasted for 9 hours, and the modified SBF was refreshed every 3 hours. Finally, the coated porous scaffolds were rinsed in ion free water and desiccated and kept in a vacuum dryer before use. 


\section{Characterization of PLGA and $\mathrm{nHA}$ coated PLGA scaffolds}

The surfaces of the scaffolds were observed, using a field emission scanning electron microscope (FE-SEM, S-4800, Hitachi High-Technologies Co, Tokyo, Japan) operated at $1 \mathrm{kV}$, after sputtered coating with gold. The size of nHA particles were analyzed using ImageJ software (free downloaded from http://imagej.nih.gov/ij). Mass fraction of HA coatings on PLGA porous scaffold $\left(\varphi_{\mathrm{w}, \mathrm{HA}}\right)$ was determined by thermo gravimetric analysis (TGA, Pyris 1, PerkinElmer Inc, Waltham, MA, USA). The TGA analysis was carried out from room temperature to $800^{\circ} \mathrm{C}$ at a heating rate of $20^{\circ} \mathrm{C} /$ minute in a nitrogen atmosphere at an airflow rate of $40 \mathrm{~mL} /$ minute.

The porosity of two different scaffolds was measured using the liquid displacement method with absolute ethanol., ${ }^{5,18}$ Briefly, a dry porous scaffold was weighed as $m_{\text {scffl,dry }}$. Ethanol was fully pressed into the pores of the scaffold by vacuum and then weighed as $m_{\text {scffl,wet }}$. The $\rho_{\mathrm{C}_{2} \mathrm{H}_{5} \mathrm{OH}}$ and $\rho_{\mathrm{PLGA}}$ were the density of absolute ethanol and PLGA, respectively. The porosity of the scaffold was calculated as follows:

$\varphi=\frac{\left(m_{\text {scffd,wet }}-m_{\text {scffd,dry }}\right) / \rho_{\mathrm{C}_{2} \mathrm{H}_{5} \mathrm{OH}}}{m_{\text {scffd,dry }} / \rho_{\text {PLGA }}+\left(m_{\text {scffd,wet }}-m_{\text {scffd,dry }}\right) / \rho_{\mathrm{C}_{2} \mathrm{H}_{5} \mathrm{OH}}} \times 100 \%$

While for HA coated PLGA scaffolds, we first determined the mass of nHA by TGA detection, then obtained the weight fraction of nHA over PLGA plus nHA, $f_{\mathrm{w}, \mathrm{HA}}$. The theoretical porosity was then calculated by:

$$
\begin{gathered}
\varphi=\frac{\left(m_{\text {scffd,wet }}-m_{\text {scffd,dry }}\right) / \rho_{\mathrm{C}_{2} \mathrm{H}_{5} \mathrm{OH}}}{\left(1-f_{\mathrm{w}, \mathrm{HA}}\right) m_{\text {scffd,dry }} / \rho_{\mathrm{PLGA}}+f_{\mathrm{w}, \mathrm{HAp}} m_{\text {scffd,dry }} / \rho_{\mathrm{HA}}} \times 100 \% \\
\quad+\left(m_{\text {scffd,wet }}-m_{\text {scffd,dry }}\right) / \rho_{\mathrm{C}_{2} \mathrm{H}_{5} \mathrm{OH}}
\end{gathered}
$$

The scaffolds used in this study were discs of $\phi 4 \mathrm{~mm} \times 3 \mathrm{~mm}$ for the in vitro experiments and cylinders of $\phi 4 \mathrm{~mm} \times 15 \mathrm{~mm}$ for the in vivo experiments.

\section{Isolation and culture of autologous BMSCs}

All animal experiments in this study were performed after permission granted by the institutional animal care committee of The Fourth Military Medical University. The BMSCs were prepared as previously described.$^{20}$ Briefly, three New Zealand rabbits aged between 2.5 and 3 months were anesthetized using $1 \%$ pentobarbital sodium $(30 \mathrm{mg} / \mathrm{kg})$. Then, a 16-gauge medullo-puncture needle was acupunc- tured into the right posterior iliac crest of each animal. Afterwards, a $10 \mathrm{~mL}$ syringe containing $1 \mathrm{~mL}$ physiological saline (within $1000 \mathrm{U}$ heparin) was connected to the needle. When about $4 \mathrm{~mL}$ of bone marrow was harvested, the needle was moved from the rabbit with continuous shaking of the syringe to avoid cruor. ${ }^{21}$ Five $\mathrm{mL}$ of bone marrow (containing $1 \mathrm{~mL}$ heparin) was collected in a sterile $15 \mathrm{~mL}$ test tube. The harvested bone marrow was immediately put into centrifuge in a test tube and concentrated at $2000 \mathrm{rpm}$ for 20 minutes. The precipitates were resuspended in a Dulbecco's modified Eagle medium (DMEM, Hyclone, Logan, UT, USA) containing 10\% fetal bovine serum (FBS, Gibco, New York, $\mathrm{NY}, \mathrm{USA}), 100 \mathrm{U} / \mathrm{ml}$ penicillin, and $100 \mathrm{mg} / \mathrm{L}$ streptomycin, and then were cultured at $37^{\circ} \mathrm{C}$ in a humidified atmosphere of $5 \% \mathrm{CO}_{2}$. The medium was changed every two days. The third-passage cell subcultures were used in the following experiments.

\section{Seeding of BMSCs}

Scaffolds (virgin PLGA and nHA coated PLGA) were sterilized by soaking into $75 \%$ ethanol for 24 hours. Before BMSC seeding, the scaffolds were washed in phosphate buffered saline (PBS, pH 7.2-7.6) three times and DMEM for another three times. BMSCs were digested with trypsin/ ethylene diaminetetraacetic acid (EDTA) to produce a cell suspension, concentrated by centrifugation at $1200 \mathrm{rpm}$ for 12 minutes and seeded onto the top of scaffolds at $2 \times 10^{6}$ cells. The scaffolds with seeding cells were put into 6 -well plates and incubated at $37^{\circ} \mathrm{C}$ supplemented with $5 \% \mathrm{CO}_{2}$. BMSCs were subcultured with the scaffolds in the same medium for one week changing the medium every 48 hours before being used in the next experiment. ${ }^{22}$

\section{Scanning electron microscope (SEM) observation of BMSC-seeded scaffolds}

After culturing for 1-week, the specimens (virgin PLGA and nHA coated PLGA) were removed from the plates, gently washed with PBS three times, and fixed with 3\% glutaraldehyde in PBS at room temperature for 8 hours. Next, the composites were dehydrated in series of ethanol solutions ranging from $50 \%$ to $99 \%$, air dried, and sputter-coated with a 60-nm layer of gold. ${ }^{23}$ Composites were viewed with SEM (S-3400N, Hitachi High-Technologies Co).

\section{Cytotoxicity of extracted liquid}

The scaffolds were extracted with DMEM, and the cytotoxicity of the extract liquid was examined using a 3-(4, 5-dimethylthiazoyl-2-yl)-2, 5-diphenyltertrazolium bromide 
(MTT) assay. First, composites were soaked in the culture medium at $37^{\circ} \mathrm{C}$ and $5 \% \mathrm{CO}_{2}$ for 48 hours. The leachate was then diluted to $1 / 2$ and $1 / 4$ concentration. BMSCs (third passage) were seeded in 96-well plates $\left(1 \times 10^{4}\right.$ cells $)$ in $200 \mu \mathrm{L}$ DMEM at $37^{\circ} \mathrm{C}$ and $5 \% \mathrm{CO}_{2}$. The medium was replaced with $200 \mu \mathrm{L} /$ well of the leachate (original, 1/2, and 1/4 concentration) 48 hours later. Each type of leachate was placed in 6 wells. Twenty-four hours later, $20 \mu \mathrm{L}$ MTT (Sigma-Aldrich Co, St Louis, MI, USA) was added to each well; the incubation was kept for 4 hours until the medium was replaced with $150 \mu \mathrm{L}$ of dimethyl sulfoxide (DMSO). The plates were surged at $200 \mathrm{rpm}$ for 15 minutes to dissolve formazan crystals and were measured using Infinite M200 (Tecan Group Ltd, Mannedorf, Switzerland) at wavelength of $492 \mathrm{~nm}$. The absorbance value of plates represented the relative cell viability.

\section{Proliferation of cells in scaffolds}

Cell proliferation was also indirectly detected by the MTT assay. Two kinds of scaffolds were cut into segments (discs; $\phi 4 \mathrm{~mm}$ in diameter and $3 \mathrm{~mm}$ in thickness). Each group had 9 scaffolds. Cell seeding was performed as described in the previous subsections. The segments that combined with BMSCs were placed into a 96-well plate. The wells with no segments were used as control. The segments were incubated at $37^{\circ} \mathrm{C}$ at $5 \% \mathrm{CO}_{2}$ for 3,5 , or 7 days. At each time point, three samples from each group were detected by MTT assay. The solution of the MTT was transferred to another 96-well plate for the detection. The relative proliferation ratio (RPR) was calculated by the absorbance of the test well over the absorbance of the control well.

\section{Implantation surgery}

We used a total of 45 white, male, 5-month old, New Zealand rabbits weighing $2.5-2.9 \mathrm{~kg}$ in this study. Animals were anesthetized with an injection into ear marginal veins. Methods used to create the segmental defect model were according to the literature. ${ }^{24}$ A $15 \mathrm{~mm}$ bone defect was created in the middle shaft of the right radius of each rabbit under the anesthesia with $1 \%$ pentobarbital sodium $(30 \mathrm{mg} / \mathrm{kg})$. It is recognized that the critical size for a bone defect is $15 \mathrm{~mm}$ in length: without bone implantation or replacement, the bone defect cannot be self-repaired. ${ }^{25}$

Thirty-six rabbits were randomly divided into two groups. Eighteen rabbits were put into each group depending on the different scaffolds used. In Group A, the nHA coated PLGA scaffolds with BMSCs were put into the defect; in Group B, the virgin PLGA scaffolds with BMSCs were used. Every rabbit received a scaffold with its own BMSCs. The implants were placed into the defect areas without any fixation. The defects of the remaining 9 rabbits were left empty without scaffolds and were used as the blank control group. When the implanting surgery was done, muscles, fascia, and skin were closed separately in routine ways. Antimicrobial therapy was provided with penicillin for 3 days. After 4, 8, and 12 weeks, six animals each from Groups A and B, and three animals from the blank control group were sacrificed by an overdose intravenous injection of pentobarbital. After euthanasia, radius containing the implant and its associated ulna were removed and examined by X-ray, Micro-computerized tomography (Micro-CT), and histological evaluations.

\section{Fluorochrome labeling}

Sequential fluorochrome markers Calcein $(30 \mathrm{mg} / \mathrm{kg}$, SigmaAldrich) and Tetracycline (30 mg/kg, Sigma-Aldrich) were administered via intramuscular injection 2 weeks and 3 days before the animals were sacrificed, respectively. Twelve weeks later, pathological sections were applied for fluorescence analysis using an epifluorescence microscopy. The speed of new bone formation was measured by the length between two labels over time (11 days).

\section{Radiological examination}

The harvested radius-ulna specimens were examined by $\mathrm{X}$-ray to evaluate new bone formation in the animals (X-ray source: $46 \mathrm{kV}, 50 \mathrm{~mA}$; exposure time: 0.14 seconds). Radiographs were scored using a semiquantitative scoring system according to the literature (Table 1$){ }^{26}$

\section{Micro-CT examination}

Specimens were placed in the sample holder supplied by the Micro-CT company and scanned using a Micro-CT (eXplore Locus SP, GE Healthcare Bio-Sciences) after they were fixed in $80 \%$ ethanol for 24 hours. Each tomogram was acquired at projections of $1024^{2}$ pixels of about 1600 at an X-ray source voltage of $80 \mathrm{kV}$ and a beam current of $200 \mathrm{~mA}$. The scanning rotation angular was $180^{\circ}$, with an angular increment of $0.40^{\circ}$.

Table I Scoring system for semiquantitative evaluation of radiographs

\begin{tabular}{ll}
\hline Criterion & Score \\
\hline No obvious bone regeneration & 0 \\
Less than 50\% bone regeneration & 1 \\
More than 50\% bone regeneration & 2 \\
Almost fused & 3 \\
Fused-not full thickness & 4 \\
Fused-full thickness & 5 \\
\hline
\end{tabular}


All specimens were reconstructed using three-dimension analysis software (Microview; GE Healthcare Bio-Sciences). The percentage of bone volume to total volume and bone mineral density (BMD) were determined at a threshold of 2000 for the expression of bone tissue.

\section{Biomechanical test}

In the biomechanical test, six normal radii (without surgery) were set as the control group, and six specimens from each group after 12 weeks of bone defect healing were tested. The biomechanical properties of the samples were characterized using a three-point bending test using a universal testing machine (Instron, London, UK). The span was $40 \mathrm{~mm}$ which was larger than the defect area of $15 \mathrm{~mm}$ and the loading speed was $1 \mathrm{~mm} /$ minute. The loading continued until sample fracture. The biomechanical properties of bones were determined by ultimate loading $(\mathrm{N})$ and fracture stress $\left(\mathrm{N} / \mathrm{mm}^{2}\right)$. The data were reported as mean plus standard error of the mean.

\section{Histology analysis}

Specimens from bone defect were fixed in an $80 \%$ ethanol solution for 1-week. Then, the dehydration of the specimens was performed by putting them in a graded ethanol series (70\%-100\%) within 2 weeks. Finally, the specimens were transferred into a methylmethacrylate (MMA) solution that polymerized at $37^{\circ} \mathrm{C}$ for 24 hours. Two or three slices of pathological sections (about $50 \mu \mathrm{m}$ in thickness) were prepared along the long axis of radius at the central region using a Leica saw microtome (SP1600, Wetzlar, Germany). Afterwards, the sections were stained by $1.2 \%$ trinitrophenol and 1\% acid fuchsin (Van Gieson staining) and observed using a light microscope (DM6000B, Leica Microsystems). In order to carry out histomorphometry analysis, the sections with bone tissue were pseudocolored using Adobe Photoshop CS6 software (Adobe Systems Incorporated, San Jose, CA, USA) at the same threshold and analyzed using the Image-Pro Plus system (Media Cybernetics, Silver Spring, MD, USA). The new bone formation was quantified from the pixels representing bone tissue. The total area was regarded as the implanted bone site $(\phi 4 \mathrm{~mm} \times 15 \mathrm{~mm})$. The rate of new bone formation was determined by the percentage of the bone area over the total implant area ([bone area/total area] $\times 100 \%$ ).

\section{Statistical analysis}

All quantitative data were analyzed with SPSS 14.0 software (IBM Corporation, Armonk, NY, USA). Statistical comparisons were carried out using the Student's $t$-test. A $P$-value less than 0.05 was considered statistically significant.

\section{Results}

\section{Preparation and modification of scaffolds}

PLGA porous scaffolds were prepared at room temperature using the molding/particle leaching method, and a part of the virgin PLGA scaffolds was further modified by coating the interior pore surfaces with nHA. The exterior sizes of both the nHA coated PLGA scaffolds and the virgin PLGA scaffolds were $4 \mathrm{~mm}$ in diameter and $15 \mathrm{~mm}$ in height. The presence of HA in the PLGA scaffold was confirmed by TGA first by detecting the weight loss with increases in temperature (data not shown). The organic component of PLGA burned up gradually between the temperature range of $200^{\circ} \mathrm{C}-500^{\circ} \mathrm{C}$, and a dramatic weight loss occurred between $300^{\circ} \mathrm{C}-400^{\circ} \mathrm{C}$. The weight of PLGA scaffold was almost stable above $500^{\circ} \mathrm{C}$, indicating that the PLGA had totally burned out. In contrast, the inorganic component of HA could not burn out till $800^{\circ} \mathrm{C}$. The residue weight versus the initial weight gave the weight fraction of nHA, $f_{\mathrm{w}, \mathrm{HA}}$, which read $14.1 \%$.

We further used the liquid replacement approach to detect scaffold porosity. The volume porosities of the two kinds of scaffolds were both around $90 \%(91.2 \% \pm 0.3 \%$ for virgin PLGA scaffolds and $90.8 \% \pm 0.4 \%$ for nHA coated PLGA scaffolds, without significant difference). We can determine that nHA is likely to be well coated on the pore walls of scaffolds, as nHA coating has a relative high mass amount but no significant influences on porosity of the scaffold.

The interior pore surfaces of scaffolds were further directly visualized by FE-SEM, as shown in Figure 1. The nHA coated PLGA and PLGA scaffolds exhibited a

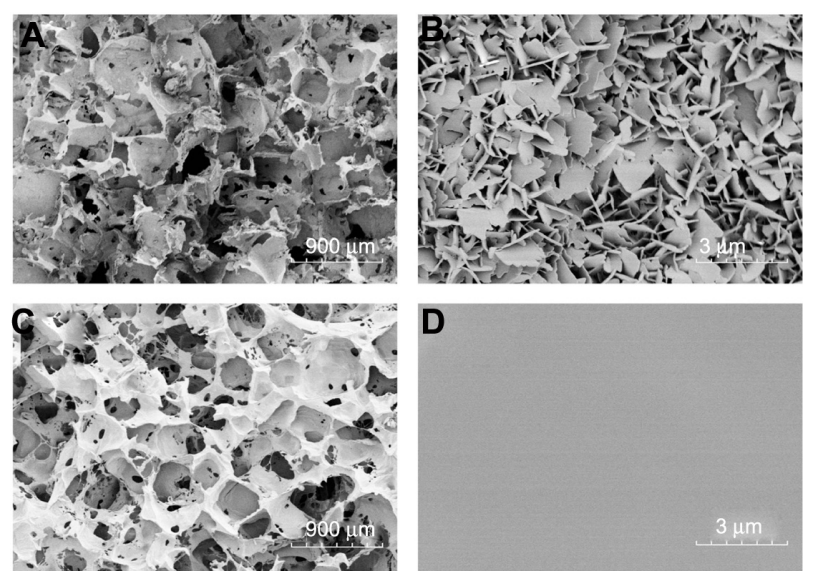

Figure I FE-SEM images of the pore surfaces of scaffolds. nHA coated PLGA scaffold (A and B); virgin PLGA scaffold (C and D).

Notes: The nHA coated PLGA and PLGA scaffolds exhibited a homogeneously interconnected 3D structure at low magnification ( $\mathbf{A}$ and $\mathbf{C}$ ). At high magnification, the nHA coated PLGA scaffolds had an interior surface coated by lamella like hydroxyapatite (B), while the surfaces of PLGA scaffolds were relatively smooth (D). Abbreviations: FE-SEM, field emission scanning electron microscope; $\mathrm{nHA}$, nano-hydroxyapatite; PLGA, poly (lactic-co-glycolic acid). 
homogeneously interconnected 3D structure at low magnification (Figure $1 \mathrm{~A}$ and $\mathrm{C}$ ). The pore diameters were $300-450 \mu \mathrm{m}$ and the pores were intercommunicated. High magnification SEM pictures of the scaffolds revealed differences between the two kinds of scaffolds, and the nHA coated PLGA scaffolds had a lamella structure of hydroxyapatite (Figure 1B). The thickness of the hydroxyapatite slice was about $50 \mathrm{~nm}$, the width was 400-1000 nm, and the hydroxyapatite slices were closely packed. In contrast, the surfaces of PLGA scaffolds were relatively smooth (Figure 1D).

\section{Cell adhesion on scaffold pores}

We also observed cells on the interior surfaces. After culture of BMSCs for 7 days, more cells were found on the surface of the nHA coated PLGA scaffolds (Figure 2A and B), and extracellular matrix (ECM) was actively secreted. In contrast, the number of BMSCs on the PLGA scaffolds were relatively lower (Figure $2 \mathrm{C}$ and $\mathrm{D}$ ) than on the nHA coated PLGA scaffolds, as was ECM secretion. Hence, BMSCs grow better on the nHA modified surfaces of the nHA coated PLGA scaffolds than on the smooth surfaces of the virgin PLGA scaffolds.

\section{MTT assay}

MTT assay was used to measure the cytotoxicity of the extracted liquid of porous scaffolds. As shown in Figure 3A, the extracted liquids from both scaffolds did not display apparent cytotoxicity. The RPR of BMSCs in the extracted liquids from nHA coated PLGA scaffolds was $102.2 \%$,
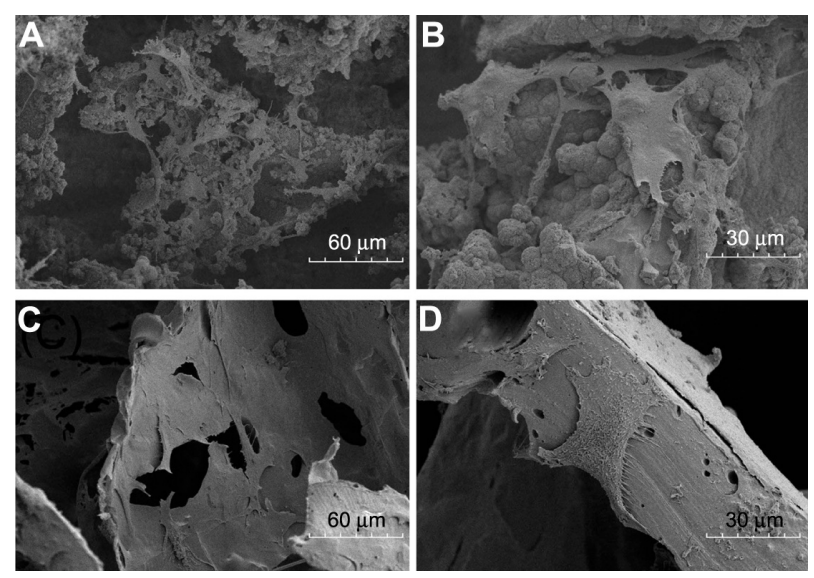

Figure 2 SEM images of BMSCs on scaffold surfaces for 7 days. nHA coated PLGA (A and $\mathbf{B})$; virgin PLGA scaffold (C and $\mathbf{D})$.

Notes: More cells were found on the surface of the nHA coated PLGA scaffolds (A) than the PLGA scaffolds (C). On the nHA coated PLGA scaffolds, BMSCs stretched their pseudopodium and actively secreted ECM (B).

Abbreviations: ECM, extracellular matrix; BMSCs, bone marrow stromal cells; nHA, nano-hydroxyapatite; PLGA, poly (lactic-co-glycolic acid); SEM, scanning electron microscope.
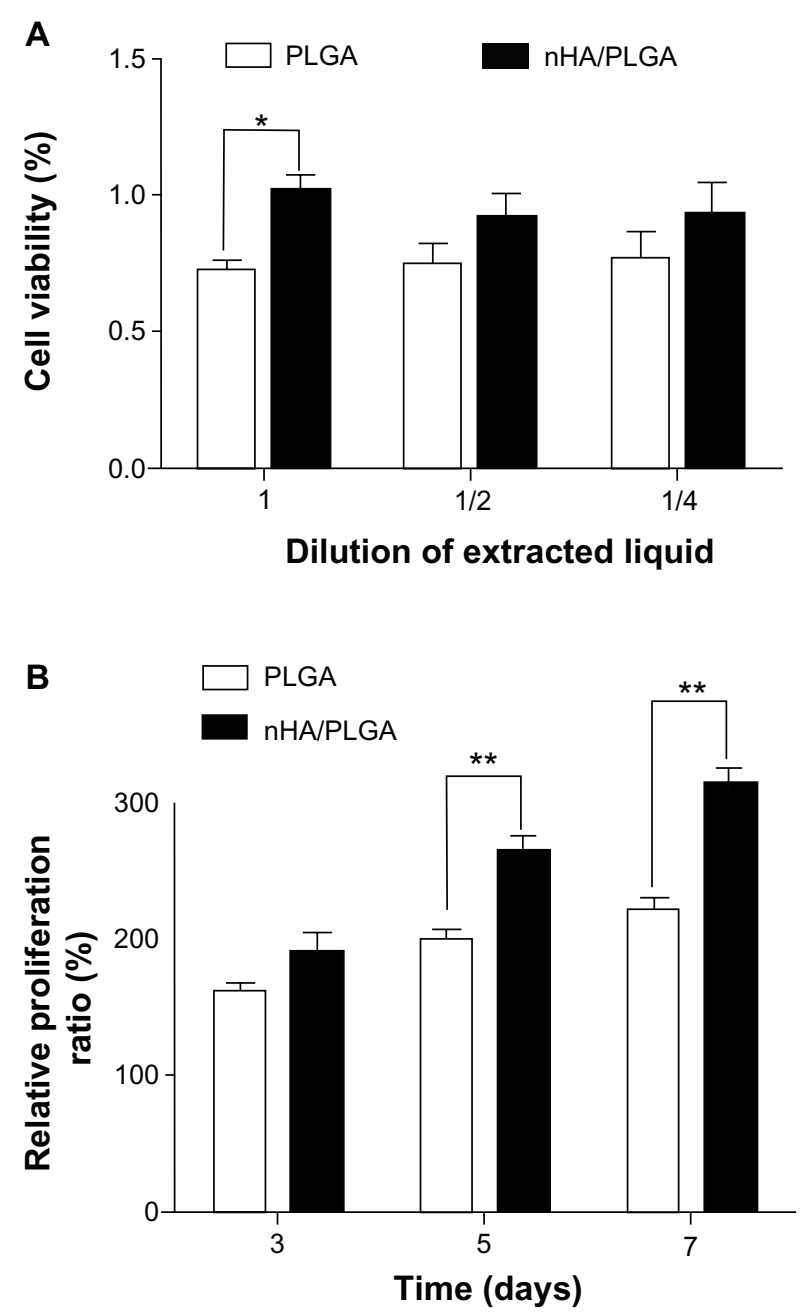

Figure 3 Cytotoxicity analysis and RPR of BMSCs on scaffolds. Cytotoxicity analysis of BMSCs survived in material leaching liquor using MTT assay; $n=3$ (A). RPRs of BMSCs growing on PLGA and nHA coated PLGA scaffolds after 3, 5, and 7 days in vitro (B).

Notes: $* \mathrm{p}<0.05$ and $* * \mathrm{P}<0.01$ compared to the other group, $\mathrm{n}=6$.

Abbreviations: BMSC, bone marrow stromal cells; MTT, 3-(4, 5-dimethylthiazoyl2-yl)-2, 5-diphenyltertrazolium bromide; nHA, nano-hydroxyapatite; PLGA, poly (lactic-co-glycolic acid); RPR, relative proliferation ratio.

$92.7 \%$, and $93.9 \%$ for the pristine extracted liquids, $1 / 2$ concentration, and 1/4 concentration, respectively. In comparison, the RPR for PLGA scaffolds was only $72.7 \%$ for pristine liquids, $74.9 \%$ for the $1 / 2$ concentration, and $77.1 \%$ for the $1 / 4$ concentration, respectively. The difference between the RPRs of BMSCs in the pristine extracted liquids from nHA coated PLGA scaffolds and PLGA scaffolds was statistically significant $(P=0.0009)$.

MTT assays were also used to assess the RPR of BMSCs on the different scaffolds. As shown in Figure 3B, after 3 days of culture, the RPR of nHA coated PLGA scaffolds was $24.2 \%$ higher than that of PLGA scaffolds, although the difference was not significant $(P=0.0863)$. The differences of RPR on day $5(P=0.0006)$ and day $7(P=0.0001)$ were 
statistically significant. On day five, the RPR of nHA coated PLGA scaffolds was $56.9 \%$ higher than that of PLGA scaffolds, and it was $80.8 \%$ higher on day seven. This result was in accordance with the SEM findings on day seven.

\section{Radiological examination}

$\mathrm{X}$-ray examinations were performed to evaluate the position of the scaffolds and the development of bone regeneration within the defects (Figure 4A). The bone defect was over the critical size and thus cannot be self-repaired, as shown in the blank group. In Groups A and B, bone formation started 4 weeks after surgery. The area of bone defect with a $15 \mathrm{~mm}$ length was filled with bony callus, which was visually
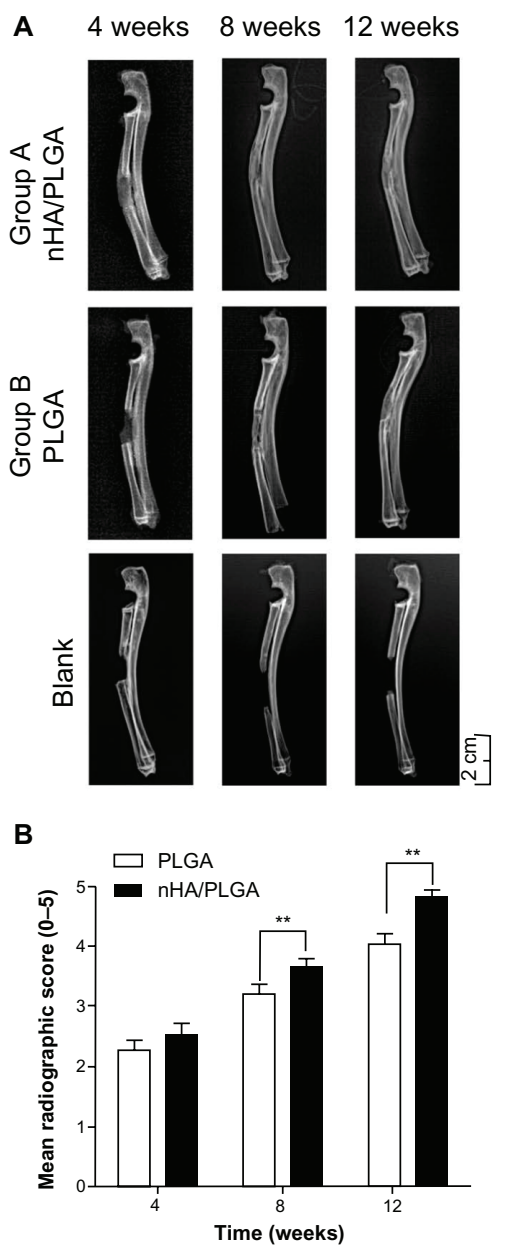

Figure 4 X-ray images and analysis of radius restoration. (A) In the blank group, the bone defect was not repaired. In Groups A and B, the initial bone defect was filled with bony callus 4 weeks after surgery, as reflected by the cloudy shadow in the X-ray photo. The area of shadow in Group A was larger than in Group B (4 weeks). Cortical bone began to form in Group A (8 weeks). Bone formation was observed and bony union was recovered after 12 weeks in Group A. While in Group B cortical bone was difficult to see. Scale Bar: $2 \mathrm{~cm}$. (B) Radiological scores demonstrating superior repairing of the defect in the group of $\mathrm{nHA}$ coated PLGA scaffolds compared to the group of virgin PLGA scaffolds.

Note: ${ }^{* * P}<0.05$

Abbreviations: nHA, nano-hydroxyapatite; PLGA, poly (lactic-co-glycolic acid). observed as the cloudy shadow on the X-ray photo. The area of shadow in Group A (4 weeks) was larger than that in Group B (4 weeks), indicating better bony callus formation in the nHA coated PLGA group. After 8 weeks, the bony callus began to be absorbed, and cortical bone began to form in Group A. While the cortical bone was hard to see in Group B, Group A exhibited significant bone formation and bony union after 12 weeks.

The radiographic scoring of the X-ray was measured by a blinding method with the criterion listed in Table 1. The results are presented in Figure 4B. The assessment of repair status defects demonstrated a statistically significant improved bridging of the defects with nHA coated PLGA scaffolds compared to PLGA scaffolds $(P=0.0010$ after 8 weeks; $P=0.0010$ after 12 weeks).

\section{Micro-CT}

Micro-CT images further confirmed the radiology results. While the 4 week images in Figure 5 reflect the porotic stage, the cortical bone formation stage is displayed in the 12 week images. A whole bone cortex was seen in Group A. In contrast, the group of virgin PLGA scaffold was not.

A
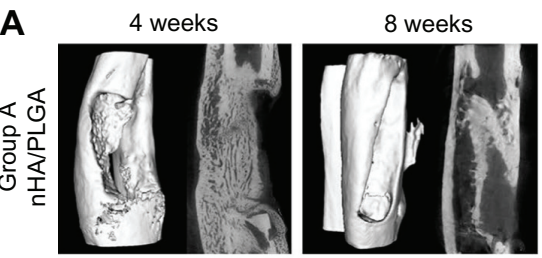
12 weeks
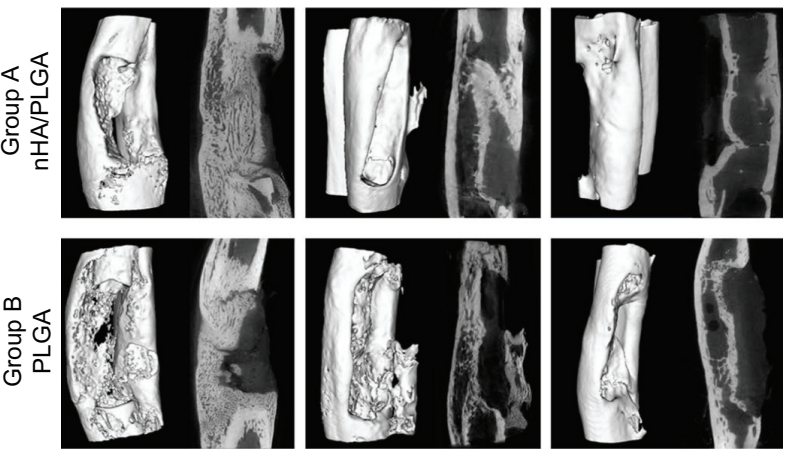

B

C
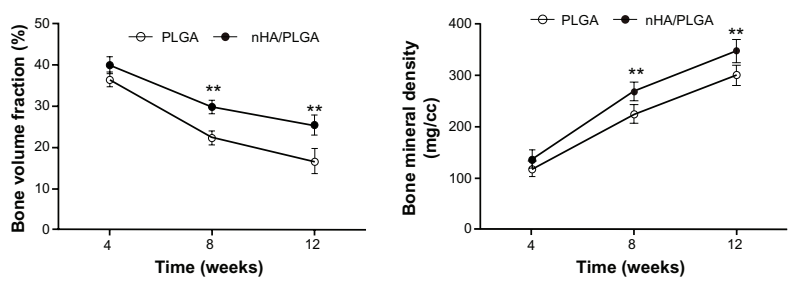

Figure 5 Micro-CT images and analysis of new bone formation. (A) Porotic stage was displayed on the 4th week images in both groups. In the 8th week, images of Group A reveal the beginnings of cortical bone formation. Good cortical bone formation was displayed on images from the 12th week in Group A. The virgin PLGA scaffold (Group B) did not form a whole bone cortex. (B) The ratio of bone volume/total volume (\%) in indicated groups and time points. $* * P<0.0 \mathrm{I}$, compared to another group at the same time point. (C) The bone mineral density in each group compared to another group at the same time point. $* * p<0.01$, compared to another group at the same time point.

Abbreviations: $\mathrm{nHA}$, nano-hydroxyapatite; PLGA, poly (lactic-co-glycolic acid). 
By employing 3D analysis software (Microview), a region of interest (ROI), a cylinder with a diameter of $6 \mathrm{~mm}$ and a length of $15 \mathrm{~mm}$, was defined. Total Volume (TV) was the volume of the ROI. The ROI was centered in the defect area with the purpose of calculating the bone volume (BV) in the ROI by setting the threshold of 2000 for bone tissue manually. These results are shown in Figure 5B. At every time point, the bone volume fraction (BVF, BVF=BV/TV) in the nHA coated PLGA group was higher than in the PLGA group, and a significant difference was found at $8(P=0.0007)$ and 12 weeks $(P=0.004)$. Therefore, the quantity of new bone in Group A was higher than that in Group B.

When BVF declined, bone mineral density (BMD; $\mathrm{BMD}=$ bone mineral content $/ \mathrm{BV}[\mathrm{mg} / \mathrm{cc}]$ ) increased. The BMDs of both groups at 12 weeks were higher than those at 4 weeks. At every time point, the BMD of Group A was higher than that of Group B.

\section{Biomechanical results}

The results of biomechanical testing are shown in Table 2 . In general, the nHA coated PLGA scaffolds enhanced the mechanical properties of the restored new bones as evidenced by a higher ultimate loading compared to the virgin PLGA scaffolds. However, compared to the normal radius (without surgery), the biomechanics of the repaired radius by nHA coated scaffolds were not sufficiently strong. The fracture stresses of Group A and Group B were not significantly different.

\section{Fluorochrome labeling}

Fluorescent labeling was detected in the regenerated bone at 12 weeks in specimens from Group A (Figure 6A) and Group B (Figure 6B). The two images were both taken with an optical microscope at a magnification of 200. The results showed faster new bone regeneration in nHA modified PLGA scaffolds. The speed of new bone formation in Group A was $3.7 \pm 0.3 \mu \mathrm{m} /$ day, while in Group B it was $1.9 \pm 0.2 \mu \mathrm{m} /$ day; a difference that was statistically significant $(P=0.005)$.

\section{Histology analysis}

To evaluate the tissue response to the implanted scaffolds and the defect healing progress, we performed histological examination using Van Gieson staining. As shown in Figure 7A, callus was

Table 2 Results of biomechanical tests after 12 weeks

\begin{tabular}{lcrr}
\hline & Normal radius & Group A & Group B \\
\hline Ultimate loading $(\mathrm{N})$ & $119.0 \pm 7.2$ & $84.5 \pm 5.8$ & $6 \mathrm{I} \pm 1.4$ \\
Fracture stress $\left(\mathrm{N} / \mathrm{mm}^{2}\right)$ & $6.4 \pm 0.7$ & $4.9 \pm 0.8$ & $4.2 \pm 0.7$ \\
\hline
\end{tabular}

Notes: Group A, the nHA coated PLGA scaffolds; Group B, the virgin PLGA scaffolds.
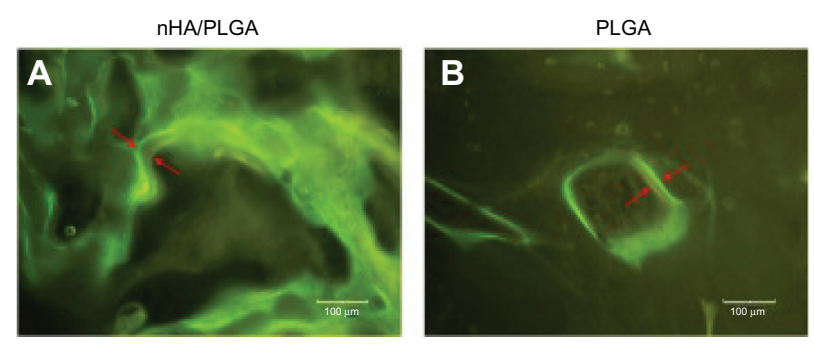

Figure 6 Fluorescent labeling of images to indicate the fronts of new bone formation within II days.

Notes: In vivo staining was carried out by fluorochrome markers Calcein and Tetracycline at 14 and 3 days, respectively, before the animals were sacrificed. The speed of new bone formation was calculated by the length between two labels/days ( $\mu \mathrm{m} /$ day).

Abbreviations: nHA, nano-hydroxyapatite; PLGA, poly (lactic-co-glycolic acid).

eugenic in the specimens at 4 weeks in both Groups A and B. There was a difference between the two groups which appeared after 8 weeks, the callus masses were absorbed in Group A. Although bone cortex began to form in Group B, the process proceeded slowly. nHA coated PLGA scaffolds led to a better result at the 12th week, with complete bone cortex regeneration; even the medullary cavity was reformed.

The percentage of bone formation (bone area/total area) in the initial defect site during the 4th week in Group A $(40 \% \pm 2 \%)$ was similar to in Group B $(36 \% \pm 2 \%)$. At later time points, however, the percentage of bone formation in Group A was significantly higher than of Group B: at the 8th week, the percentages were $30 \% \pm 2 \%$ in Group $A$ and $22 \% \pm 2 \%$ in Group B $(P=0.0031)$; after 12 weeks, the percentages were $25 \% \pm 2 \%$ in Group A and $17 \% \pm 3 \%$ in Group B $(P=0.0060)$. These results are summarized in Figure 7B.

\section{Discussion}

Ideal scaffolds for tissue engineering have the following qualities: good biocompatibility, appropriate physical structure, appropriate degradation rate, and good processibility. Owing to these features, porous PLGA scaffolds have been widely used in tissue engineering, including for the treatment of bone defects. ${ }^{6,27-30}$ While PLGA scaffolds have many advantages, their limited abilities regarding osteoconduction and osteoinduction hinder their application in bone tissue engineering. The surface characteristics of biomedical implants play an important role in implanting and eventual osteointegration. ${ }^{31}$ Therefore, in this study, we fabricated PLGA scaffolds and further modified their interior surfaces by nHA coating.

PLGA and HA are polymeric and inorganic materials, respectively. The compositing technique used to mix PLGA and HA must deal with a compatibility problem, and this influences the basic mechanical and processing properties 

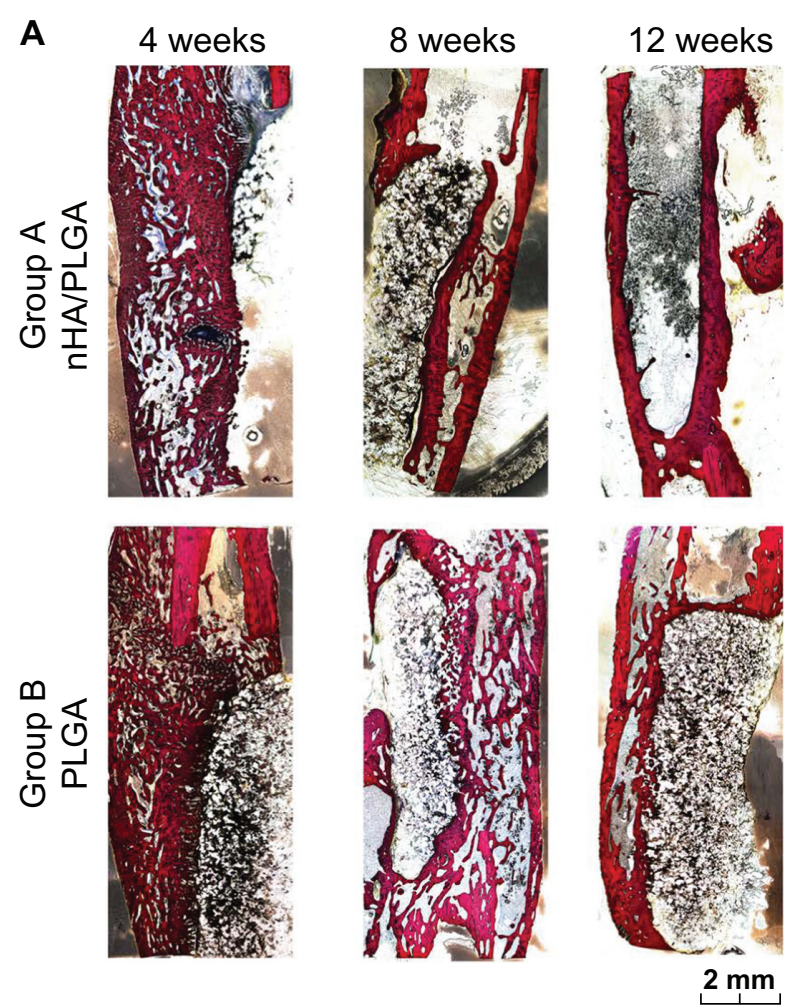

B

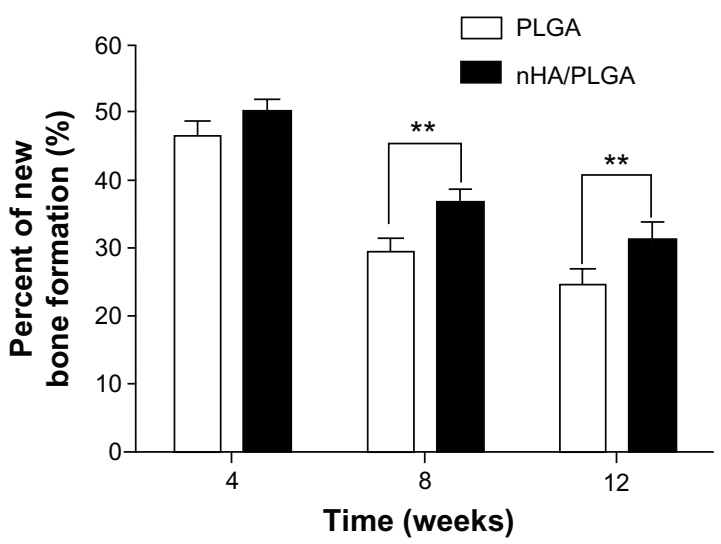

Figure 7 Histological images and histomorphometric analysis of bone formation. (A) Merged histological micrographs of sections upon van Gieson staining. The tissue stained in red is the newly formed bone with visible cell nuclei. The tissue stained in yellow/black indicates the fibrous tissue and undegraded material. After 4 weeks, callus were eugenic in Groups A and B. After 8 weeks, a callus mass was absorbed in Group A, while significant callus still remained in Group B. After 12 weeks, nHA coated PLGA scaffolds showed a better result. As depicted in the images, whole bone cortex was regenerated in Group A at the 12th week, and the medullary cavity was reformed. (B) Histomorphometric analysis.

Note: $* * P<0.01$, compared to another group at the same time point.

Abbreviations: nHA, nano-hydroxyapatite; PLGA, poly (lactic-co-glycolic acid).

of the well described PLGA scaffolds. To overcome these issues, we employed a coating technique by maintaining the PLGA scaffolds as the main matrix.

A naive HA coating on the PLGA surface also poses some difficulties. First, due to the incompatibility between organic and inorganic materials, the coated layer might not tightly bind to the main matrix. Second, the long time period required for precipitation in water leads to partial degradation of the PLGA and thus the properties of the eventual scaffolds are not well controlled.

In the present study, we employed an improved approach for combining plasma treatment and precipitation of $\mathrm{nHA}$ in SBF to modify the interior surfaces of PLGA porous scaffolds. Plasma treatment can generate reactive sites, and thus the combination of plasma treatment and SBF precipitation guarantees tight binding of the nHA layer to the PLGA matrix. Another characteristic of our surface modification technique is that we did not use normal SBF. Our modified SBF distinguishes itself as multiple folds of the saturation concentration and preset $\mathrm{pH}$ before precipitation, with release parameters described in the Materials and methods section. Therefore, we spent only 9 hours modifying the interior pore surfaces of our scaffolds. This less modification time is very beneficial, especially for reducing unexpected degradation of PLGA in the coating process in aqueous medium.

Our in vitro experiments indicated that the nHA layer was stable and the modified PLGA scaffolds showed better biocompatibility than PLGA scaffolds. The cytotoxicity experiments (Figure 3A) suggested that the two composites in this study were not toxic for BMSCs and can be used in cell seeding and bone defect restoring. The results of cell proliferation experiments demonstrated that the nHA coated PLGA scaffolds promoted faster cell proliferation (Figure 3B). Cell adhesion on the scaffold surface indicates the biocompatibility of biomaterials to a certain extent ${ }^{32}$ by changing the cytoskeleton, the material surface can influence cellular reactions. ${ }^{33}$ The results of SEM for BMSCs composites and scaffolds indicated that cells had better morphology and living conditions on nHA coated PLGA scaffolds (Figure 1).

Surface chemistry and physics can significantly regulate cell behaviors. ${ }^{34-38}$ The improved bioactivity of nHA coated PLGA surfaces in the present study may be attributed to the following: first, the modified surfaces are rough and contain a lot of calcium and phosphorus, which are biomimetic and helpful for cell adhesion, migration, proliferation, and bone matrix formation. In bone tissue engineering, the BMSCs defined as seed cells, can disintegrate the skeleton of the scaffolds by secreting proteases in the early stages of bone remodeling. Seeding BMSCs on the scaffolds can produce some structural proteins before they die, which may enhance the biomimetic abilities in the local structure of the scaffolds. Therefore, the migrated mesenchymal stem cells can enter into the interior of the scaffolds. Second, the modified surface is a nanostructure, on which cells can adhere and differentiate better. ${ }^{39}$ Finally, the HA coating on the scaffolds 
can induce a microenvironment, particularly an alkalization in the medium change, which has a positive influence on cell metabolism, ${ }^{40}$ especially with respect to PLGA scaffolds and acidic degradation products.

Our in vivo experiments further demonstrated the good efficacy of nHA coated PLGA scaffolds for bone defect repairing. The radiographic results (Figure 4A) suggested that the hydroxyapatite layer can enhance bone regeneration. Both Micro-CT and histological evaluation further confirmed this hypothesis. The formation of new cortex and recanalization of the marrow cavity was even observed via inspection of Group A (12 week). The BVF of the new bone was descending as shown by Micro-CT and histology analysis. Though BVF was reduced, BMD was increased. Further analysis using the pathological sections indicated that when the callus turned into cortical bone, the BVF and BMD were inversed. In order to calculate the speed of new bone formation, bone labeling was used to note the deposition of new bone. In Figure 6, the green line indicates the labeling by Calcein, and the yellow line indicates the labeling by Tetracycline; the bone labeling was detected in both groups. The longer the distance between the green and yellow lines, the faster the new bone formation was occurring. A large number of calluses formed at the beginning, and the calluses were gradually absorbed and reconstructed into the cortical bone.

$\mathrm{HA}$ is a natural component of bone. It can promote the proliferation and metabolism of osteoblasts on the nanoscale because of its similar structure to natural bone. ${ }^{41,42}$ Therefore, HA is a beneficial composition for use in bone defect repairing. ${ }^{43}$ However crystalline HA degrades over a long period in vivo and a large amount of undegraded HA may thus hinder or slow complete bone reconstruction. The nHA particles show improved bioactivity and osteointegration over conventional HA particles in vivo. ${ }^{44}$ In this study, we coated nHA tightly and rapidly onto the interior surfaces of PLGA porous scaffolds using our modified technique. The nHA coating significantly reduced the total amount of HA required without significantly reducing the pore area while producing a nanostructure on the pore surfaces. Our new and facile material technique retained the excellent processibility of PLGA porous scaffolds and also introduced the biomimetic moiety of nHA. Hence, nHA coated PLGA scaffolds combine advantages of both HA and PLGA, and are promising as a material for use in bone tissue engineering.

\section{Conclusion}

In this study, we fabricated nHA coated PLGA scaffolds using a facile approach: we first obtained virgin porous PLGA scaffolds using room temperature molding and particular leaching, and then modify the interior pore surfaces by plasma treatment and precipitation of nHA in a modified SBF solution with a very high ionic concentration and a preset medium $\mathrm{pH}$. The in vitro and in vivo experiments based on rabbit BMSCs and bone segment repairing in a rabbit model confirmed that the nHA coated PLGA scaffolds had improved biocompatibility and were more efficient for healing bone defects. These results demonstrate that the introduction of nHA coating using our improved surface modification approach can significantly improve the biocompatibility and bioactivity of PLGA scaffolds.

\section{Acknowledgments}

This work was supported by the National Key Basic Research Program of China (973 Program, No 2009CB930000).

\section{Disclosure}

The authors report no conflicts of interest in this work.

\section{References}

1. Bhatt RA, Rozental TD. Bone graft substitutes. Hand clin. 2012;28(4): 457-468.

2. Van der Stok J, Van Lieshout EM, El-Massoudi Y, Van Kralingen GH, Patka P. Bone substitutes in The Netherlands - a systematic literature review. Acta biomater. 2011;7(2):739-750.

3. Zhang ZY, Teoh SH, Chong MS, et al. Neo-vascularization and bone formation mediated by fetal mesenchymal stem cell tissue-engineered bone grafts in critical-size femoral defects. Biomaterials. 2010;31(4): 608-620.

4. Park SH, Park DS, Shin JW, et al. Scaffolds for bone tissue engineering fabricated from two different materials by the rapid prototyping technique: PCL versus PLGA. J Mater Sci Mater Med. 2012;23(11): 2671-2678.

5. Wu LB, Zhang H, Zhang JC, Ding JD. Fabrication of three-dimensional porous scaffolds of complicated shape for tissue engineering. I. Compression molding based on flexible-rigid combined mold. Tissue Eng. 2005;11(7-8):1105-1114.

6. Pan Z, Ding JD. Poly(lactide-co-glycolide) porous scaffolds for tissue engineering and regenerative medicine. Interface focus. 2012; 2(3):366-377.

7. Zhang $\mathrm{P}, \mathrm{Wu} \mathrm{H}, \mathrm{Wu} \mathrm{H}$, et al. RGD-conjugated copolymer incorporated into composite of poly(lactide-co-glycotide) and poly(L-lactide)-grafted nanohydroxyapatite for bone tissue engineering. Biomacromolecules. 2011;12(7):2667-2680.

8. Ge S, Zhao N, Wang L, et al. Bone repair by periodontal ligament stem cellseeded nanohydroxyapatite-chitosan scaffold. Int J Nanomedicine. 2012; 7:5405-5414.

9. Wang Z, Li M, Yu B, Cao L, Yang Q, Su J. Nanocalcium-deficient hydroxyapatite-poly (e-caprolactone)-polyethylene glycol-poly (e-caprolactone) composite scaffolds. Int J Nanomedicine. 2012;7: 3123-3131.

10. Cao L, Duan PG, Wang HR, et al. Degradation and osteogenic potential of a novel poly(lactic acid)/nano-sized $\beta$-tricalcium phosphate scaffold. Int J Nanomedicine. 2012;7:5881-5888.

11. Anselme K. Osteoblast adhesion on biomaterials. Biomaterials. 2000; 21(7):667-681.

12. Liu H, Webster TJ. Nanomedicine for implants: a review of studies and necessary experimental tools. Biomaterials. 2007;28(2):354-369. 
13. Santos MI, Unger RE, Sousa RA, Reis RL, Kirkpatrick CJ. Crosstalk between osteoblasts and endothelial cells co-cultured on a polycaprolactone-starch scaffold and the in vitro development of vascularization. Biomaterials. 2009;30(26):4407-4415.

14. Santos MI, Tuzlakoglu K, Fuchs S, et al. Endothelial cell colonization and angiogenic potential of combined nano- and micro-fibrous scaffolds for bone tissue engineering. Biomaterials. 2008;29(32):4306-4313.

15. Wu LB, Ding JD. Effects of porosity and pore size on in vitro degradation of three-dimensional porous poly(D,L-lactide-co-glycolide) scaffolds for tissue engineering. J Biomed Mater Res A. 2005;75A(4):767-777.

16. Wu LB, Zhang JC, Jing DY, Ding JD. "Wet-state" mechanical properties of three-dimensional polyester porous scaffolds. J Biomed Mater Res A. 2006;76A(2):264-271.

17. Zhang JC, Wu LB, Jing DY, Ding JD. A comparative study of porous scaffolds with cubic and spherical macropores. Polymer. 2005;46(13): 4979-4985.

18. Wu LB, Ding JD. In vitro degradation of three-dimensional porous poly(D,L-lactide-co-glycolide) scaffolds for tissue engineering. Biomaterials. 2004;25(27):5821-5830.

19. Kokubo T, Takadama H. How useful is SBF in predicting in vivo bone bioactivity? Biomaterials. 2006;27(15):2907-2915.

20. Bi L, Hu Y, Fan H, et al. Treatment of contaminated bone defects with clindamycin-reconstituted bone xenograft-composites. J Biomed Mater Res B Appl Biomater. 2007;82(2):418-427.

21. Tu J, Wang H, Li H, Dai K, Wang J, Zhang X. The in vivo bone formation by mesenchymal stem cells in zein scaffolds. Biomaterials. 2009;30(26):4369-4376.

22. Omae H, Mochizuki Y, Yokoya S, Adachi N, Ochi M. Augmentation of tendon attachment to porous ceramics by bone marrow stromal cells in a rabbit model. Int Orthop. 2007;31(3):353-358.

23. Mygind T, Stiehler M, Baatrup A, et al. Mesenchymal stem cell ingrowth and differentiation on coralline hydroxyapatite scaffolds. Biomaterials. 2007;28(6):1036-1047.

24. An YH, Friedman RJ. Animal models of orthopedic implant infection. J Invest Surg. 1998;11(2):139-146.

25. Mokbel N, Bou Serhal C, Matni G, Naaman N. Healing patterns of critical size bony defects in rat following bone graft. Oral Maxillofac Surg. 2008;12(2):73-78.

26. Bodde EW, Spauwen PH, Mikos AG, Jansen JA. Closing capacity of segmental radius defects in rabbits. J Biomed Mater Res A. 2008;85(1): 206-217.

27. Amini AR, Adams DJ, Laurencin CT, Nukavarapu SP. Optimally porous and biomechanically compatible scaffolds for large-area bone regeneration. Tissue Eng Pat A. 2012;18(13-14):1376-1388.

28. Henslee AM, Spicer PP, Yoon DM, et al. Biodegradable composite scaffolds incorporating an intramedullary rod and delivering bone morphogenetic protein-2 for stabilization and bone regeneration in segmental long bone defects. Acta Biomater. 2011;7(10):3627-3637.
29. Wu LB, Jing DY, Ding JD. A "room-temperature" injection molding/ particulate leaching approach for fabrication of biodegradable threedimensional porous scaffolds. Biomaterials. 2006;27(2):185-191.

30. Wang Z, Zhang Z, Zhang JC, She ZJ, Ding JD. Distribution of bone marrow stem cells in large porous polyester scaffolds. Chinese Sci Bull. 2009;54(17):2968-2975.

31. Nicula R, Lüthen F, Stir M, Nebe B, Burkel E. Spark plasma sintering synthesis of porous nanocrystalline titanium alloys for biomedical applications. Biomol Eng. 2007;24(5):564-567.

32. Asti A, Visai L, Dorati R, et al. Improved cell growth by Bio-Oss/PLA scaffolds for use as a bone substitute. Technol Health Care. 2008;16(6): 401-413.

33. Webster TJ, Siegel RW, Bizios R. Osteoblast adhesion on nanophase ceramics. Biomaterials. 1999;20(13):1221-1227.

34. Jiao Y, Liu Z, Zhou C. Fabrication and characterization of PLLAchitosan hybrid scaffolds with improved cell compatibility. J Biomed Mater Res A. 2007;80(4):820-825.

35. Peng R, Yao X, Ding JD. Effect of cell anisotropy on differentiation of stem cells on micropatterned surfaces through the controlled single cell adhesion. Biomaterials. 2011;32(32):8048-8057.

36. Pan Z, Yan C, Peng R, Zhao YC, He Y, Ding JD. Control of cell nucleus shapes via micropillar patterns. Biomaterials. 2012;33(6):1730-1735.

37. Peng R, Yao X, Cao B, Tang J, Ding JD. The effect of culture conditions on the adipogenic and osteogenic inductions of mesenchymal stem cells on micropatterned surfaces. Biomaterials. 2012;33(26):6008-6019.

38. Yao X, Peng R, Ding JD. Effects of aspect ratios of stem cells on lineage commitments with and without induction media. Biomaterials. 2013; 34(4):930-939.

39. Wang X, Yan C, Ye K, HeY, LiZH, Ding JD. Effect of RGD nanospacing on differentiation of stem cells. Biomaterials. 2013;34(12):2865-2874.

40. Busa WB, Nuccitelli R. Metabolic regulation via intracellular pH. Am J Physiol. 1984;246(4 Pt 2):R409-R438.

41. Huang J, Best SM, Bonfield W, et al. In vitro assessment of the biological response to nano-sized hydroxyapatite. J Mater Sci Mater Med. 2004;15(4):441-445.

42. Pezzatini S, Solito R, Morbidelli L, et al. The effect of hydroxyapatite nanocrystals on microvascular endothelial cell viability and functions. J Biomed Mater Res A. 2006;76(3):656-663.

43. Bernards MT, Qin C, Jiang S. MC3T3-E1 cell adhesion to hydroxyapatite with adsorbed bone sialoprotein, bone osteopontin, and bovine serum albumin. Colloids Surf B Biointerfaces. 2008;64(2):236-247.

44. Lewandrowski KU, Bondre SP, Wise DL, Trantolo DJ. Enhanced bioactivity of a poly(propylene fumarate) bone graft substitute by augmentation with nano-hydroxyapatite. Biomed Mater Eng. 2003;13(2): $115-124$.
International Journal of Nanomedicine

\section{Publish your work in this journal}

The International Journal of Nanomedicine is an international, peerreviewed journal focusing on the application of nanotechnology in diagnostics, therapeutics, and drug delivery systems throughout the biomedical field. This journal is indexed on PubMed Central,

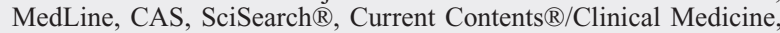

\section{Dovepress}

Journal Citation Reports/Science Edition, EMBase, Scopus and the Elsevier Bibliographic databases. The manuscript management system is completely online and includes a very quick and fair peer-review system, which is all easy to use. Visit http://www.dovepress.com/ testimonials.php to read real quotes from published authors. 